\title{
Time regularity of Lévy-type evolution in Hilbert spaces and of some $\alpha$-stable processes. ${ }^{* \dagger}$
}

\author{
Witold Bednorz Grzegorz Głowienko $^{\S} \quad$ Anna Talarczyk $^{\Uparrow}$
}

\begin{abstract}
In this paper we consider the cylindrical càdlàg property of a solution to a linear equation in a Hilbert space $H$, driven by a Levy process taking values in a possibly larger Hilbert space $U$. In particular, we are interested in diagonal type processes, where processes on coordinates are functionals of independent $\alpha$-stable symmetric processes. We give the equivalent characterization in this case. We apply the same techniques to obtain a sufficient condition for existence of a càdlàg version of stable processes described as integrals of deterministic functions with respect to symmetric $\alpha$-stable random measures with $\alpha \in[1,2)$.
\end{abstract}

Keywords: càdlàg and cylindrical càdlàg trajectories; path properties; Ornstein-Uhlenbeck processes; linear evolution equations; Lévy noise; stable processes.

MSC2020 subject classifications: Primary 60H15, Secondary 60G52; 60G17.

Submitted to ECP on August 2, 2020, final version accepted on May 14, 2021.

\section{Introduction}

We first consider a linear equation in a Hilbert space $H$ given by $d X_{t}=A X_{t} d t$, $X_{0}=x$, where $A$ is a generator of a $C_{0}$ semigroup $(S(t))_{t \geqslant 0}$ on $H$. Obviously, the solution can be represented as $X_{t}=S(t) x$. We may perturb the linear equation by a Lévy process $Z=\left(Z_{t}\right)_{t \in T}$ which takes values in $U$, where $U$ is a Hilbert space $H \subset U$. It leads to the following equation of evolution with Lévy noise

$$
d X_{t}=A X_{t} d t+d Z_{t}, \quad t \in T=[0, a], \quad X_{0}=0, \quad a>0 .
$$

Note that if $U=H$, then once again (1.1) can be easily solved

$$
X_{t}=\int_{0}^{t} S(t-s) d Z_{s}, \quad X_{0}=0 .
$$

If $H \subset U$ then the solution exists in a weaker form which we discuss in a special case below. The equations of the form (1.1) were considered e.g. in [8], [10], [9], [6], [7]. One may wonder whether there exists a càdlàg version of $X=\left(X_{t}\right)_{t \in T}$ in $H$. This problem is described in Liu Zhai [6] and the answer is that if such a modification exists, then $Z$ takes

\footnotetext{
${ }^{*}$ Research supported in part by National Science Centre, Poland, grant 2016/21/B/ST1/01489.

${ }^{\dagger}$ Research supported in part by National Science Centre, Poland, grant 2016/23/B/ST1/00492.

${ }^{\ddagger}$ University of Warsaw, E-mail: wbednorz@mimuw. edu.pl

§University of Warsaw, E-mail: g291623@mimuw.edu.pl

"University of Warsaw, E-mail: annatal@mimuw.edu.pl
} 
values in $H$. However, in some cases $X$ may take values in $H$, even though the space, in which the noise lives, is larger. One may wonder what other kinds of regularity one may expect. In [9] several other notions of càdlàg property have been introduced, which are weaker than càdlàg in $H$. In the present paper we focus on the cylindrical càdlàg property. We consider the case when both $Z$ and the equation (1.1) is of a diagonal form.

In the this paper we consider only the diagonal case with negative diagonal operator $A$ and diagonal Lévy-type process $Z$, which is a much simpler question. Namely, let $\left(e_{n}\right)_{n=1}^{\infty}$ be an orthonormal and complete basis in $H$, we assume that for any $n=1,2, \ldots$ vector $e_{n}$ belongs to the domain of $A$ and $A e_{n}=-\gamma_{n} e_{n}$ with $\gamma_{n}>0$. Moreover, assume that $Z_{t}=\sum_{n=1}^{\infty} Z_{t}^{(n)} e_{n}$, where $Z^{(n)}$ are real-valued independent symmetric Lévy processes without Gaussian part and with Lévy measures $\mu_{n}$, respectively. Note that, in general the sum defining $Z$ may not converge in $H$, but in some larger space Hilbert $U$. By the solution to the diagonal type evolution equation we mean the process

$$
X_{t}=\sum_{n=1}^{\infty} X_{t}^{(n)} e_{n}, \quad t \in[0, a]
$$

where

$$
d X_{t}^{(n)}=-\gamma_{n} X_{t}^{(n)} d t+d Z_{t}^{(n)}, \quad X_{0}^{(n)}=0, \quad n=1,2, \ldots
$$

The process $X$ takes values in $H$ if and only if the series $\sum_{n=1}^{\infty}\left(X_{t}^{(n)}\right)^{2}$ converges in probability (and therefore almost surely, thanks to independence). One can express this condition in terms of Lévy measures $\mu_{n}$ (see Proposition 2.6 in [9]). An important example considered in literature is when $Z^{(n)}=\sigma_{n} L^{(n)}$, where $L^{(n)}$ are independent standard symmetric $\alpha$-stable Lévy processes and $\sigma_{n} \geqslant 0$. This will be referred to as the $\alpha$-stable case. In this case, the condition for $X$ to take values in $H$ is

$$
\sum_{n=1}^{\infty} \frac{\sigma_{n}^{\alpha}}{1+\gamma_{n}}<\infty
$$

The proof of the fact can be also found in [10]. Note that in [10] it was assumed that $\gamma_{n} \rightarrow \infty$, hence condition (1.4) was written with $\gamma_{n}$ in the denominator, instead of our $1+\gamma_{n}$, but the proof without the assumption that $\gamma_{n}$ tend to infinity is essentially the same (cf. Proposition 4.2 in [10]).

Moreover, by the Liu Zhai result [6] we know that in the $\alpha$-stable case, there exists a càdlàg version of $X=\left(X_{t}\right)_{t \in T}$ in $H$ if and only if $Z$ takes values in $H$, which is equivalent to

$$
\sum_{n=1}^{\infty} \sigma_{n}^{\alpha}<\infty
$$

However, the intriguing situation is when (1.5) fails - which means that $Z$ has values beyond the space $H$. The question is whether we can still expect some regularity of $X$. The regularity we analyze in this paper is the existence of a cylindrical càdlàg modification.

According to the Definition 1.1 in [9] an $H$-valued process $X$ is cylindrical càdlàg if for any $z \in H$ the real valued process

$$
Y_{t}=\left\langle z, X_{t}\right\rangle=\sum_{n=1}^{\infty}\left\langle z, e_{n}\right\rangle X_{t}^{(n)}, \quad t \geqslant 0
$$

has a càdlàg modification. Note that if $X$ is a cylindrical càdlàg, then for any finite set of vectors $z_{1}, z_{2}, \ldots, z_{n} \in H$ the process

$$
\left(\left\langle z_{1}, X\right\rangle,\left\langle z_{2}, X\right\rangle, \ldots,\left\langle z_{n}, X\right\rangle\right)
$$


has a càdlàg modification and hence the property indicates weak regularity of $X$ as of a process in high dimensions. There are some partial results towards the question discussed in the extensive paper [9] (note that there are discussed many forms of regularity). However, the results in [9] do not completely cover even the basic question of $Z^{(n)}$ that are $\alpha$-stable, where $\alpha \in(1,2)$. We do propose an approach which in particular covers the question formulated as Question 4 in [9]. It should be mentioned that the case of $\alpha \in(0,1]$ was completely solved in [7].

As it will be proved, our approach works in the much more general setting of diagonal type evolution equations implying a nice sufficient condition for the cylindrical càdlàg property for all diagonal type equations.

The process $Y$ of (1.6) clearly depends on $z \in H$, however since $z$ will be fixed we do not stress this dependence in the notation. Moreover, even though $X$ does not take values in $H$, it is possible that the process $Y$ is well defined and we may consider the problem of the existence of its càdlàg modification.

The key idea in the proof is to use the Poissonian representation of Lévy processes and an application of a result of [3] concerning suprema of Bernoulli processes. In this approach it is important that the Lévy processes are symmetric.

In the last part of the paper we show the usefulness of our method beyond the evolution equations. Namely, we give sufficient conditions for existence of càdlàg modifications of stable processes of the form

$$
X_{t}=\int_{E} f(t, x) M(d x) \quad t \in[0, a],
$$

where $M$ is a symmetric $\alpha$-stable random measure and $f$ is a deterministic function satisfying appropriate integrability conditions. See Section 5 and Theorem 5.1 below. It is worth stressing that our condition also works in the case $\alpha \in(1,2)$, which seems to be a difficult one.

The paper is organized as follows. In Section 2 we introduce some notation and representations of the process $Y$ given by (1.6). In Section 3 we discuss a necessary condition for existence of a càdlàg modification of the process $Y$. In Section 4 we provide a sufficient condition. Finally, in Section 5 we discuss the problem of càdlàg modification of stable processes of the form (1.7).

\section{Representation of solution}

For the sake of simplicity we assume that $T=[0,1]$. As we have explained the solution to the evolution equation has the form (1.2). Suppose that $Z^{(n)}=\sigma_{n} L^{(n)}$, where $\sigma_{n} \geqslant 0$ and $L^{(n)}, n=1,2, \ldots$ are independent symmetric Lévy processes without Gaussian component and with Lévy measures $\nu_{n}$, respectively. That is, $L_{t}^{(n)}$ has characteristic function of the form

$$
\mathbf{E} e^{i \theta L_{t}^{(n)}}=\exp \left\{-t \int_{\mathbb{R}}(1-\cos (\theta y)) \nu_{n}(d y)\right\},
$$

where $\nu_{n}$ is a symmetric Borel measure on $\mathbb{R}$, satisfying $\nu_{n}(\{0\})=0$ and

$$
\int_{\mathbb{R}}\left(y^{2} \wedge 1\right) \nu_{n}(d y)<\infty .
$$

Such processes have a càdlàg modification, and in the sequel we will always assume that $L^{(n)}, n=1,2, \ldots$ are càdlàg. As described in the introduction we assume that $A$ is a diagonal operator, and for an orthonormal basis $\left(e_{n}\right)_{n}$ of $H$ we have $A e_{n}=-\gamma_{n} e_{n}$, with 
$\gamma_{n}>0$. Then (1.3) reads as

$$
X_{t}^{(n)}=\int_{0}^{t} \exp \left(-\gamma_{n}(t-s)\right) \sigma_{n} d L_{s}^{(n)} .
$$

It is well known that the jump times and sizes of $L^{(n)}$ are points of a Poisson random measure, with intensity measure $\ell \otimes \nu_{n}$, where $\ell$ is the Lebesgue measure on $\mathbb{R}_{+}$. We denote this random measure by $\pi_{n}$. Thus

$$
L_{t}^{(n)}=\lim _{\delta \rightarrow 0} \int_{0}^{t} \int_{|y| \geqslant \delta} y \pi_{n}(d s, d y),
$$

where the limit is a.s. Moreover, on a subsequence $\delta_{n} \searrow 0$ fast enough the convergence is a.s. uniform on bounded intervals (see e.g. Theorem 6.8 in[8]). Note that here we do not need to compensate, since $\nu_{n}$ are symmetric.

Also, due to symmetry $\pi_{n}$ can be represented as a sum of Dirac measures

$$
\pi_{n}=\sum_{k} \delta_{\left(t_{n, k},\left(\tilde{\varepsilon}_{n, k} y_{n, k}\right)\right)}
$$

where $\left(t_{n, k}, y_{n, k}\right)$ are points of a Poisson random measure with intensity $\ell \otimes \mu_{n}$ with $\mu_{n}(B)=2 \nu_{n}\left(B \cap \mathbb{R}_{+}\right)$, which will be denoted here by $\pi_{n}^{+}$, and $\tilde{\varepsilon}_{n, k} k=1,2, \ldots$ are i.i.d. Rademacher random variables. In this setting the process $L^{(n)}$ at time $t_{n, k}$ has a jump of absolute value $y_{n, k}$ and $\operatorname{sign} \tilde{\varepsilon}_{n, k}$, i.e.

$$
\Delta L_{t_{n, k}}^{(n)}=\tilde{\varepsilon}_{n, k} y_{n, k}
$$

For $n=1,2, \ldots$ the corresponding Poisson random measures $\pi_{n}^{+}$and random signs are independent.

An important example is when $L^{(n)}$ are symmetric $\alpha$-stable processes with $\alpha \in(0,2)$. In this case it is well known that

$$
\nu_{n}(d y)=\frac{C_{\alpha}}{|y|^{\alpha+1}} d y
$$

Here $C_{\alpha}>0$ is a constant that standardizes $L^{(n)}$, so that

$$
\mathbf{E} e^{i \theta L_{t}^{(n)}}=e^{-t|\theta|^{\alpha}}
$$

We fix $z \in H$ and consider existence of a càdlàg modification of

$$
Y_{t}=\left\langle X_{t}, z\right\rangle=\sum_{n=1}^{\infty} Y_{t}^{(n)}=\sum_{n=1}^{\infty}\left\langle z, e_{n}\right\rangle X_{t}^{(n)}, \quad t \in[0,1] .
$$

where $X^{(n)}$ are given by (2.1), and $Y_{t}^{(n)}=\left\langle z, e_{n}\right\rangle X_{t}^{(n)}$.

Under a weak assumption the sum $\sum_{n} Y_{t}^{(n)}$ converges a.s. for all $t \in T=[0,1]$, we explain it below. Each of the variables $Y_{t}^{(n)}, t \in T$ can be represented in terms of the Poisson random measure $\pi_{n}$ as

$$
Y_{t}^{(n)}=\lim _{\delta \rightarrow 0+} \sum_{k: y_{n, k} \geqslant \delta} b_{n} \varepsilon_{n, k} y_{n, k} e^{-\left(t-t_{n, k}\right) \gamma_{n}} 1_{t_{n, k} \leqslant t},
$$

where $b_{n}=\left|\sigma_{n}\left\langle z, e_{n}\right\rangle\right|, \varepsilon_{n, k}=\tilde{\varepsilon}_{n, k} \operatorname{sgn}\left(\left\langle z, e_{n}\right\rangle\right)$ and $t_{n, k}, y_{n, k}, \tilde{\varepsilon}_{n, k}, n=1,2, \ldots, i=1,2, \ldots$ are as above. 
Proposition 2.1. For any $t \in[0,1]$ the sum on the right hand side of (2.3) converges almost surely if and only if

$$
\psi(\theta):=\sum_{n=1}^{\infty} \int_{0}^{t} \int_{\mathbb{R}}\left(1-\cos \left(\theta b_{n} y e^{-\gamma_{n} s}\right)\right) \nu_{n}(d y) d s<\infty, \quad \theta \in \mathbb{R}
$$

and the function $\psi$ is continuous at 0 .

Proof. By (2.4), using the form of the characteristic function of integrals with respect to a Poisson random measure (see e.g. Theorem 6.6 in [8]) we have

$$
\mathbf{E} e^{i \theta Y_{t}^{(n)}}=\exp \left\{-\int_{0}^{t} \int_{\mathbb{R}}\left(1-\cos \left(\theta b_{n} y e^{-\gamma_{n}(t-s)}\right)\right) \nu_{n}(d y) d s\right\}, \quad \theta \in \mathbb{R} .
$$

$Y_{t}^{(n)}$ are independent, hence almost sure convergence of the series (2.3) is equivalent to its convergence in law and the result follows.

In particular, if $L^{(n)}$ are standard symmetric $\alpha$-stable Lévy processes, then recalling (2.2) we have

$$
\int_{0}^{t} \int_{\mathbb{R}}\left(1-\cos \left(\theta b_{n} y e^{-\gamma_{n} s}\right)\right) \nu_{n}(d y) d s=\int_{0}^{t}|\theta|^{\alpha}\left(b_{n} e^{-\gamma_{n} s}\right)^{\alpha} d s=|\theta|^{\alpha} b_{n}^{\alpha} \frac{1-e^{-\alpha \gamma_{n} t}}{\alpha \gamma_{n}} .
$$

Since $\frac{1-e^{-x}}{x}$ for $x>0$ is bounded from above and below by $\frac{1}{1+x}$ multiplied by a constant, we see that the series (2.3) converges almost surely for any $t \in[0,1]$ if and only if

$$
\sum_{n=1}^{\infty} \frac{b_{n}^{\alpha}}{1+\gamma_{n}}<\infty
$$

It is clear that each of the processes $Y^{(n)}$ is càdlàg. Thus, using (2.4) we can use the following representation of $Y$

$$
Y_{t}=\left\langle z, X_{t}\right\rangle=\sum_{n} Y_{t}^{(n)}=\sum_{n} \sum_{k} b_{n} \varepsilon_{n, k} y_{n, k} e^{-\left(t-t_{n, k}\right) \gamma_{n}} 1_{t_{n, k} \leqslant t}, \quad t \in T,
$$

The sum over $k$ is understood as $\lim _{\delta \rightarrow 0} \sum_{k: y_{n, k} \geqslant \delta} \ldots$. We are ready to discuss the convergence of $\sum_{n} Y_{t}^{(n)}, t \in T$.

The main idea we follow is that $\left(Y_{t}\right)_{t \in T}$ can be split into two parts according to whether $b_{n} y_{n, k} \geqslant 1$ or $b_{n} y_{n, k}<1$. The first part is a finite sum of càdlàg processes and in the second the series with respect to $n$, converges uniformly in $L^{1}$, thus there is a subsequence on which the convergence is a.s. uniform on $T$, hence the limit is càdlàg.

\section{Necessary condition}

Recall (2.6) and (2.5). The next theorem provides a necessary condition for $Y$ to have a càdlàg modification. This result follows from Theorem 3.4 of [7], but, as it is short, we will also present its proof, to have a full picture of our problem.

Theorem 3.1. If $Y$ has a càdlàg modification, then for any $\varepsilon>0$ we have

$$
\sum_{n=1}^{\infty} \nu_{n}\left(\left[\frac{\varepsilon}{b}, \infty\right)\right)<\infty .
$$

Example 3.1. (Cf. Corollary 3.5 in [7]). If $L^{(n)}$ are independent standard symmetric $\alpha$-stable Lévy processes and the process $Y$ has a càdlàg modification, then

$$
\sum_{n} b_{n}^{\alpha}<\infty
$$


Proof of Theorem 3.1. We argue by contradiction. Suppose that (3.1) does not hold for some $\varepsilon>0$ and that $Y$ has a càdlàg modification $\tilde{Y}$. Fix any $n$ and denote:

$$
Y_{t}^{(n, \varepsilon)}=\sum_{k: y_{n, k} \geqslant \varepsilon} b_{n} \varepsilon_{n, k} y_{n, k} e^{-\left(t-t_{n, k}\right) \gamma_{n}} 1_{t_{n, k} \leqslant t}, \quad t \geqslant 0 .
$$

Then the processes

$$
\tilde{Y}_{t}-Y_{t}^{(n, \varepsilon)}, t \geqslant 0, \quad \text { and } \quad Y_{t}^{(n, \varepsilon)}, \quad t \geqslant 0
$$

are càdlàg and they are independent (independence follows from the fact that $\pi_{n}$ is independently scattered). Moreover, $Y^{(n, \varepsilon)}$ has jumps at jump times of the Poisson process $\pi_{n}([0, t] \times\{y:|y| \geqslant \varepsilon\}), t \geqslant 0$. Therefore, with probability one, the sample paths of the two processes defined in (3.3) must have jumps at different times. Hence, with probability one, whenever $Y^{(n)}$ has a jump of size $\geqslant \varepsilon$, then $\tilde{Y}$ has a jump of equal size and sign. Notice also, that

$$
\left|\Delta Y_{s}^{(n)}\right|=b_{n}\left|\Delta L_{s}^{(n)}\right|
$$

where, for a càdlàg process $Z$ we denote $\Delta Z_{s}=Z_{s}-Z_{s-}$.

We will show that if (3.1) does not hold then, with probability one, there are infinitely many $n$, such that $L^{(n)}$ has a jump of size $\geqslant \varepsilon / b_{n}$. Moreover, all $L^{(n)}$ are independent, hence they jump at different times. Consequently, by the argument above, this implies that $\tilde{Y}$ must have an infinite number of jumps of size $\geqslant \varepsilon$ on $[0,1]$, and therefore cannot be càdlàg. This is a contradiction. $u>0$

Let $\xi^{(n)}$ denote the maximal jump of $L^{(n)}$ on $[0,1] ; \xi^{(n)}=\sup _{s \leq 1}\left|\Delta L_{s}\right|$. Clearly, for

$$
\mathbf{P}\left(\xi^{(n)}<u\right)=\mathbf{P}\left(\pi^{(n)}([0,1] \times\{y:|y| \geqslant u\})=0\right)=\exp \left(-\nu_{n}(\{y:|y| \geqslant u\})\right) .
$$

Hence

$$
\begin{gathered}
\sum_{n} \mathbf{P}\left(b_{n} \xi^{(n)} \geqslant \varepsilon\right)=\sum_{n} \mathbf{P}\left(\xi^{(n)} \geqslant \frac{\varepsilon}{b_{n}}\right) \\
=\sum_{n}\left(1-\exp \left(-2 \nu_{n}\left(\left[\frac{\varepsilon}{b_{n}}, \infty\right)\right)\right)\right. \\
\geqslant e^{-1} \sum_{n} \min \left\{2 \nu_{n}\left(\left[\frac{\varepsilon}{b_{n}}, \infty\right), 1\right\}=\infty\right.
\end{gathered}
$$

where the last equality is a consequence of the assumption of the opposite of (3.1). As $\xi^{(n)}$ are independent, the Borel Cantelli lemma implies that with probability 1 there are infinitely many $n$ such that $L^{(n)}$ has a jump of size at least $\varepsilon / b_{n}$.

\section{Sufficient condition}

We now discuss sufficient conditions for existence of càdlàg modification of $Y$.

Theorem 4.1. Assume that there exists $\varepsilon>0$ such that (3.1) is satisfied, and additionally that

$$
\sum_{n=1}^{\infty} b_{n}^{2} \int_{b_{n}|y| \leq \varepsilon}|y|^{2} \nu_{n}(d y)<\infty
$$

Then $Y$ has a càdlàg modification.

Before we go to the proof of the theorem we make several observations: 
Remark 4.1. The assumptions of Theorem 4.1 may be also written in the form

$$
\sum_{n=1}^{\infty} \int_{\mathbb{R}}\left(\left|b_{n} y\right|^{2} \wedge 1\right) \nu_{n}(d y)<\infty
$$

thus our result is stronger than Theorem 3.8 in [7], where $\left|b_{n} y\right|$ appeared with power 1 instead of the square.

Example 4.2. If $L^{(n)}$ are independent standard symmetric $\alpha$-stable Lévy processes with $\alpha \in(0,2)$ then (3.1) and (4.1) both reduce to

$$
\sum_{n} b_{n}^{\alpha}<\infty
$$

Hence by Theorems 3.1 and 4.1 (4.2) is a necessary and sufficient condition for $Y$ to have a càdlàg modification. This strengthens the result of [7] (Theorem 3.9) which was only proved there for $\alpha<1$.

Corollary 4.3. Assume (1.4). Then $X=\left(X_{t}\right)_{t \in T}, T=[0,1]$ has cylindrical càdlàg property if and only if

$$
\sum_{n=1}^{\infty} \sigma_{n}^{\frac{2 \alpha}{2-\alpha}}<\infty
$$

Proof. Recalling the definition of $b_{n},(4.2)$ is equivalent to

$$
\sum_{n}\left|\left\langle z, e_{n}\right\rangle \sigma_{n}\right|^{\alpha}<\infty
$$

For $X$ to have the cylindrical càdlàg property, (4.2) has to be satisfied for any $z \in H$. If (4.3) holds then (4.4) is satisfied by Hölder's inequality. In order to prove that (4.3) must hold we first use Baire's theorem. For $N \geqslant 1$ we define

$$
D_{N}=\left\{z \in H: \sum_{n}\left|\left\langle z, e_{n}\right\rangle \sigma_{n}\right|^{\alpha} \leqslant N\right\} .
$$

Since clearly (4.4) implies that $\bigcup_{N} D_{N}=H$, we derive from Baire's theorem that there must exist $N$ and a ball $B\left(z_{0}, r\right)$ in $H$ such that $B(z, r) \subset D_{N}$. Consequently,

$$
\sup _{z \in B(0,1)} \sum_{n}\left|\left\langle z, e_{n}\right\rangle \sigma_{n}\right|^{\alpha}<\infty .
$$

On the other hand, considering equality in Hölder's inequality one can find a sequence of $z_{N} \in B(0,1)$ such that

$$
\sum_{n}\left|\left\langle z, e_{n}\right\rangle \sigma_{n}\right|^{\alpha}=\left(\sum_{n=1}^{N}\left|\sigma_{2}\right|^{\frac{2 \alpha}{2-\alpha}}\right)^{\frac{2-\alpha}{2}} .
$$

If (4.3) fails, the latter expression tends to $\infty$, when $N \rightarrow \infty$. This is contradiction.

Note that it is possible that (1.4) is satisfied and $\sum_{n} \sigma_{n}^{\alpha}=\infty$ but (4.3) is satisfied. This means that in this case the process $X$ is not $H$-càdlàg but it is cylindrically càdlàg, and for which the process $Z$ of (1.1) does not have values in $H$.

Proof of Theorem 4.1. As in the proof of Theorem 3.1 let $\xi^{(n)}$ denote the maximal size of a jump of $L^{(n)}$ on $[0,1]$. Then, by (3.4) and an elementary estimate $1-e^{-x} \leq x$ we have that

$$
\sum_{n} \mathbf{P}\left(b_{n} \xi^{(n)} \geqslant \varepsilon\right)<\infty
$$


Borel Cantelli lemma and the fact that each $L^{(n)}$ is càdlàg imply that there are only a finite number of $y_{n, k}$ such that $b_{n} y_{n, k} \geqslant \varepsilon$.

Instead of $Y$ it is therefore enough to consider the process

$$
Y_{t}^{(\varepsilon)}:=\sum_{n=1}^{\infty} Y_{t}^{(n, \varepsilon)}, \quad t \geqslant 0
$$

where

$$
Y_{t}^{(n, \varepsilon)}=\lim _{\delta \rightarrow 0} \sum_{i: \delta \leq y_{n, k}<\varepsilon} b_{n} \varepsilon_{n, k} y_{n, k} e^{-\gamma_{n}\left(t-t_{n, k}\right)} 1_{t \geqslant t_{n, k}},
$$

since the difference between $Y$ and $Y^{(n, \varepsilon)}$ is a finite sum of càdlàg processes. Note that

$$
Y_{t}^{(n, \varepsilon)}=\sigma_{n}\left\langle z, e_{n}\right\rangle \int_{0}^{t} e^{-\gamma_{n}(t-s)} d L_{s}^{(n, \varepsilon)}
$$

where $L_{t}^{(n, \varepsilon)}=L_{t}-\sum_{s \leq t: b_{n}\left|\Delta_{s} L\right| \geqslant \varepsilon} \Delta L_{s}$. Each of the processes $Y^{(n, \varepsilon)}$ is càdlàg.

Moreover, observe that thanks to (4.1) the process

$$
L^{(\varepsilon)}=\sum_{n=1}^{\infty} \sigma_{n}\left\langle z, e_{n}\right\rangle L^{(n, \varepsilon)}
$$

is well defined and the sum converges in $L^{2}$ in the supremum norm on $[0,1]$, since $L^{(n, \varepsilon)}$ are independent martingales and

$$
\sum_{n=1}^{\infty} \mathbf{E}\left(L_{1}^{(n, \varepsilon)}\right)^{2}=\sum_{n=1}^{\infty} b_{n}^{2} \int_{b_{n}|y| \leq \varepsilon}|y|^{2} \nu_{n}(d y)<\infty
$$

by assumption (4.1). Therefore $L^{(\varepsilon)}$ is càdlàg.

The problem thus reduces to showing that

$$
L_{t}^{(\varepsilon)}-Y_{t}^{(\varepsilon)}=\sum_{n}\left(L_{t}^{(n, \varepsilon)}-Y_{t}^{(n, \varepsilon)}\right), \quad t \geqslant 0
$$

has a càdlàg modification.

We will show that with probability one the series in (4.7) converges a.s. in the supremum norm and in the topology $J_{1}$. The property implies the existence of a càdlàg modification of the limit. Since we could not find the right reference we give a short proof below for the sake of completeness.

Lemma 4.4. Suppose that real processes $\left(\eta_{t}^{(n)}\right)_{t \in T}, T=[0,1]$ are independent and càdlàg. Moreover, suppose that for any $\varepsilon>0$

$$
\lim _{N \rightarrow \infty} \sup _{n \geqslant m \geqslant N} \mathbf{P}\left(\left\|\sum_{k=m}^{n} \eta^{(k)}\right\|_{\infty}>\varepsilon\right)=0 .
$$

Then, for any $t \in[0,1]$ the process $\eta_{t}=\sum_{n=1}^{\infty} \eta_{t}^{(n)}$ has a càdlàg modification. More precisely, $\sum_{n=1}^{\infty} \eta^{(n)}$ converges a.s. in the Skorohod $J_{1}$ topology to some $\bar{\eta}$ which is the càdlàg modification of $\eta$. Moreover, the series $\sum_{n=1}^{\infty} \eta^{(n)}$ also converges uniformly.

Remark 4.5. Note that the space $D([0,1])$ equipped with the supremum norm is not separable, so we cannot follow the usual approach for separable Banach spaces. In fact we even do not know whether $\omega \rightarrow \sum_{n=1}^{m} \eta_{t}^{(n)}$ is a random variable with values in $D([0,1])$ with respect to the $\sigma$-field generated by the supremum norm. 
Proof of Lemma 4.4. For $x, y \in D([0,1])$ let

$$
d(x, y)=\inf _{\lambda \in \Lambda} \max \left(\sup _{0 \leqslant s<t \leqslant 1} \frac{\log [\lambda(t)-\lambda(s)]}{t-s},\|x-y \circ \lambda\|_{\infty}\right)
$$

where $\Lambda$ is the set of nondecreasing continuous functions from [0,1] onto itself. It is known that $d$ is a metric on $D([0,1])$ inducing the Skorohod $J_{1}$ topology and such that the space $D([0,1])$ with this metric is a Polish space (see [2]). Clearly, $d(x, y) \leqslant\|x-y\|_{\infty}$, hence

$$
\sup _{n \geqslant m \geqslant N} \mathbf{P}\left(d\left(\sum_{k=1}^{n} \eta^{(k)}, \sum_{k=1}^{m-1} \eta^{(k)}\right)>\varepsilon\right) \leqslant \sup _{n \geqslant m \geqslant N} \mathbf{P}\left(\left\|\sum_{k=m}^{n} \eta^{(k)}\right\|_{\infty}>\varepsilon\right) .
$$

The space $(D([0,1]), d)$ is complete and that is why the series $\sum_{n=1}^{\infty} \eta^{(n)}$ converges in probability in this space. By Theorem 1 [5] it also converges almost surely in the metric $d$ to some $\bar{\eta}$ which is càdlàg. Moreover, a simple consequence of (4.8) is that $\left\|\eta^{(n)}\right\|_{\infty}$ converges in probability to 0 as $n \rightarrow \infty$. Therefore, by Theorem 2 of [5], the series $\eta=\sum_{n=1}^{\infty} \eta^{(n)}$ also converges a.s. in the uniform norm. Therefore, for any fixed $t \in[0,1]$ variables $\eta_{t}=\bar{\eta}_{t}$ a.s. It completes the proof.

The processes $\eta^{(n)}=L^{n, \varepsilon}-Y^{(n, \varepsilon)}$ are independent for $n=1,2, \ldots$ and càdlàg, therefore by Lemma 4.4 it suffices to prove that the supremum norms converge in $L^{2}$.

We will prove the following lemma

Lemma 4.6. There exists a universal positive constant $C$ such that for any $k \leq m$ we have

$\mathbf{E} \sup _{t \in[0,1]}\left|\sum_{n=k}^{m}\left(L_{t}^{(n, \varepsilon)}-Y_{t}^{(n, \varepsilon)}\right)\right|^{2} \leq C_{1} E\left|\sum_{n=k}^{m}\left(L_{1}^{(n, \varepsilon)}-Y_{1}^{(n, \varepsilon)}\right)\right|^{2} \leq C_{2} \sum_{n=k}^{m} b_{n}^{2} \int_{b_{n}|y| \leq \varepsilon}|y|^{2} \nu_{n}(d y)$.

By assumption (4.1) this implies the Cauchy condition for the series in (4.7). The proof of the theorem will be complete provided that we show Lemma 4.6, which we do presently.

Proof of Lemma 4.6. Denote

$$
a_{n, k}(t)=b_{n} y_{n, k}\left(1-e^{-\gamma_{n}\left(t-t_{n, k}\right)}\right)_{+} .
$$

Then for fixed $k \leq m$

$$
\sum_{n=k}^{m}\left(L_{t}^{(n, \varepsilon)}-X_{t}^{(n, \varepsilon)}\right)=\lim _{\delta \rightarrow 0+} A^{(\delta)}
$$

where for $\delta<\varepsilon$

$$
A_{t}^{(\delta)}=\sum_{n=k}^{m} \sum_{k: \delta \leq b_{n} y_{n, k}<\varepsilon} \varepsilon_{n, k} a_{n, k}(t) .
$$

In (4.10) the limit is in $L^{2}$ for any fixed $t \in[0,1]$ moreover, it is a.s. uniform on $[0,1]$ on a subsequence $\delta_{n} \searrow 0$ fast enough.

We will estimate the expectation of the supremum norm of $A^{(\delta)}$ on $[0,1]$ using a result of [3]. Observe that the double sum in (4.11) is a.s. finite and the random processes $a_{n, k}$ are nondecreasing, $a_{n, k} \leq b_{n} y_{n, k}$, moreover $\left(\varepsilon_{n, k}\right)_{n, k}$ are independent of $\left(a_{n, k}\right)_{n, k}$. The latter processes depend only on $\pi_{n}^{+}, n=1,2, \ldots$. Conditioning on $\pi_{n}^{+} n=k, \ldots, m$ and using Theorem 1 of [3] for any $u>0$ we have

$$
\mathbf{P}_{\mathcal{E}}\left(\sup _{t \in[0,1]} A_{t}^{(\delta)} \geqslant 8 u\right) \leq 53 \mathbf{P}_{\mathcal{E}}\left(A_{1}^{\delta} \geqslant u\right)
$$


Here $\mathbf{P}_{\mathcal{E}}$ is the conditional probability were we condition on all variables but $\left(\varepsilon_{n, k}\right)_{n, k}$. Taking expectation, using the identity $\mathbf{E} \xi^{2}=2 \int_{0}^{\infty} u P(|\xi| \geqslant u) d u$ and also symmetry we obtain:

$$
\begin{aligned}
& \mathbf{E} \sup _{t \in[0,1]}\left|A_{t}^{(\delta)}\right|^{2} \leq C \mathbf{E}\left|A_{1}^{(\delta)}\right|^{2} \\
& \quad=C \mathbf{E} \sum_{n=k}^{m} \sum_{k: \delta<b_{n}\left|y_{n, k}\right| \leq \varepsilon} b_{n}^{2} y_{n, k}^{2} a_{n, k}^{2}(1) \leq \mathbf{E} \sum_{n=k}^{m} \int_{\delta \leq\left|b_{n} y\right|<\varepsilon} b_{n}^{2} y^{2} \nu_{n}(d y)
\end{aligned}
$$

Letting $\delta \rightarrow 0$ we obtain (4.9).

\section{Càdlàg modification of processes expressed as integrals with respect to symmetric stable random measures.}

A large class of stable stochastic processes studied in literature are of the form

$$
X_{t}=\int_{E} f(t, x) M(d x) \quad t \in[0, a]
$$

where $a>0, M$ is an $\alpha$-stable random measure defined on some measurable space $(E, \mathscr{B})$ and $f:[0, a] \times E \mapsto \mathbb{R}$ is a measurable function on the product space, satisfying appropriate integrability conditions. See e.g. [11] for a systematic treatment of stable integrals and stable processes. In this section we discuss a sufficient condition for the process of the form (5.1) to have a càdlàg modification (and hence for local boundedness of the process). Necessary and sufficient conditions for sample boundedness of processes of the form (5.1) in the case $\alpha<1$ are known. The case $\alpha>1$ seems to be more difficult (see Chapter 10 of [11]). Some more recent results on the càdlàg property of stable integrals of the form (5.1) can be found in [4] and [1].

It turns out that our methods used in the previous section can be applied also in this setting in case where $M$ is a symmetric $\alpha$-stable random measure.

We assume that $0<\alpha<2$ and let $m$ be a $\sigma$-finite measure on a measurable space $(E, \mathscr{B})$. Let $M$ denote a symmetric $\alpha$-stable random measure on $E$ with control measure $m$. That is, if we denote by $\mathcal{E}_{0}:=\{A \in \mathscr{B}: m(A)<\infty\}$ then $(M(A))_{A \in \mathcal{E}_{0}}$ is a family of real valued random variables such that:

(i) For any $A_{1}, A_{2}, \ldots \in \mathcal{E}_{0}$ such that $A_{i} \cap A_{j}=\emptyset$ for $i \neq j$ the random variables $M\left(A_{1}\right), M\left(A_{2}\right), \ldots$ are independent. Moreover, if we also have that $m\left(\bigcup_{n=1}^{\infty} A_{n}\right)<$ $\infty$, then

$$
M\left(\bigcup_{n=1}^{\infty} A_{n}\right)=\sum_{n=1}^{\infty} M\left(A_{n}\right), \quad \text { a.s. }
$$

(ii) If $A \in \mathcal{E}_{0}$, then $M(A)$ is a symmetric $\alpha$-stable random variable with scale parameter $(m(A))^{\frac{1}{\alpha}}$, that is

$$
\mathbf{E} e^{i \theta M(A)}=\exp \left\{-m(A)|\theta|^{\alpha}\right\}, \quad \theta \in \mathbb{R} .
$$

If $f: E \mapsto \mathbb{R}$ is a measurable function such that

$$
\int_{E}|f(x)|^{\alpha} m(d x)<\infty
$$

then one can define $\int_{E} f(x) M(d x)$. This is done in the usual way, by approximating $f$ by simple functions and passing to the limit. It turns out that for integrals defined in this way we have

$$
\mathbf{E} \exp \left\{i \int_{E} f(x) M(d x)\right\}=\exp \left\{-\int_{E}|f(x)|^{\alpha} m(d x)\right\}
$$


Therefore, if $a>0, f:[0, a] \times E \mapsto \mathbb{R}$ is measurable with respect to the $\sigma$-fields $\mathscr{B}([0, a]) \otimes \mathscr{B} / \mathscr{B}(\mathbb{R})$ and such that for any $t>0$ we have

$$
\int_{E}|f(t, x)|^{\alpha} m(d x)<\infty
$$

then the process (5.1) is well defined.

Recall also, that $M(A)$ and $\int_{E} f(t, x) M(d x)$ may be constructed using a Poisson random measure. Assume that $\pi$ is a Poisson random measure on $\mathbb{R} \times E$ with intensity measure

$$
\frac{C_{\alpha}}{|z|^{1+\alpha}} d z m(d x)
$$

where $C_{\alpha}>0$ is chosen such that

$$
\int_{\mathbb{R}}(1-\cos z) \frac{C_{\alpha}}{|z|^{1+\alpha}} d z=1
$$

Then, for $A \in \mathcal{E}_{0}$

$$
M(A)=\lim _{\delta \rightarrow 0} \int_{\{z:|z|>\delta\} \times A} z \pi(d z, d x),
$$

where the limit is in probability, and a.s.

If $\delta_{n} \searrow 0$ and $E_{n} \in \mathscr{B}$ are such that $m\left(E_{n}\right)<\infty, E_{n} \subset E_{n+1}$ for all $n$ and $\bigcup_{n} E_{n}=E$, then for fixed $t$, the stable integral with respect to the stable random measure constructed above may be represented as

$$
\int_{E} f(t, x) M(d x)=\lim _{n \rightarrow \infty} \int_{\left\{z:|z|>\delta_{n}\right\} \times E_{n}} z f(t, x) \pi(d z, d x), \quad \text { a.s. }
$$

A simple, but key observation in our context is that since the Lévy measure $\frac{c_{\alpha}}{|z|^{1+\alpha}} d z$ is symmetric, the Poisson random measure $\pi$ may be written as

$$
\pi=\sum_{k} \delta_{\left(\varepsilon_{k} y_{k}, x_{k}\right)}
$$

where $\pi^{+}=\sum_{i} \delta_{\left(y_{k}, x_{k}\right)}$ is a Poisson random measure with intensity measure $\frac{2 c_{\alpha}}{y^{\alpha+1}} 1_{y>0} d y m(d x)$ and $\varepsilon_{1}, \varepsilon_{2}, \ldots$ are i.i.d Rademacher random variables independent of $\pi^{+}$.

We have the following theorem.

Theorem 5.1. Assume that $\left(X_{t}\right)_{t \in[0, a]}$ is of the form (5.2) and $f=f_{1}-f_{2}$, where the functions $f_{1}, f_{2}:[0, a] \times E \mapsto \mathbb{R}_{+}, i=1,2$ are $\mathscr{B}([0, a]) \otimes \mathscr{B} / \mathscr{B}(\mathbb{R})$ measurable and such that there exists a set $N \in \mathscr{B}, m(N)=0$ such that for any $x \in E \backslash N$ the functions $t \mapsto f_{i}(t, x)$ are càdlàg and nondecreasing, $i=1,2$. Moreover, assume that

$$
\int_{E}\left|f_{i}(a, x)\right|^{\alpha} m(d x)<\infty, \quad i=1,2 .
$$

Then the process $\left(X_{t}\right)_{t \in[0, a]}$ defined by (5.1) has a càdlàg modification.

Remark 5.1. Assumptions of Theorem 5.1 essentially mean that for any $x \in E \backslash N$ the function $t \mapsto f(t, x)$ is càdlàg and has finite variation on $[0, a]$. Moreover, this variation as a function of $x$ is in $L^{\alpha}(E, m)$.

Proof of Theorem 5.1. Let $\pi$ be a Poisson random measure of the form (5.4) and let $\delta_{n}$ and $E_{n}$ be as in (5.3). Note that $\pi$ restricted to the set $\left\{|z|: z>\delta_{n}\right\} \times E_{n}$ is such that the number of points $\left(\varepsilon_{k} y_{k}, x_{k}\right)$ in this set is Poisson with parameter $\int_{\left\{y: y>\delta_{n}\right\}} \frac{2 c_{\alpha}}{y^{1+\alpha}} d y m\left(E_{n}\right)$ 
and then all random variables $\varepsilon_{k}, y_{k}, x_{k} k=1,2, .$. are independent, $\varepsilon_{k}$ are Rademacher random variables, $y_{k}$ have law with the density proportional to $1_{\left(\delta_{n}, \infty\right)}(y) \frac{1}{y^{1+\alpha}}$ and $x_{k}$ have the law $\left.\frac{1}{m\left(E_{n}\right)} m\right|_{E_{n}}$.

Let us denote

$$
X_{t}^{(n)}=\int_{\left\{|z|>\delta_{n}\right\} \times E_{n}} z f(t, x) \pi(d z, d x)=\sum_{k: y_{k}>\delta_{n}, x_{k} \in E_{n}} \varepsilon_{k} y_{k} f\left(t, x_{k}\right) .
$$

Clearly the process $\left(X_{t}^{(n)}\right)_{t \in[0, a]}$ is càdlàg since the sum is finite and the function $t \mapsto$ $f(t, x)$ is càdlàg for any $x \in E \backslash N$. For any $t \in[0, a] X_{t}^{(n)}$ converges pointwise to $X_{t}$. Therefore, to prove the theorem it suffices to show that the processes $X^{(n)}$ converge a.s. uniformly on $[0, a]$. Moreover, writing

$$
X_{t}^{(n)}=\int_{\left\{|z|>\delta_{n}\right\} \times E_{n}} z f_{1}(t, x) \pi(d z, d x)-\int_{\left\{|z|>\delta_{n}\right\} \times E_{n}} z f_{2}(t, x) \pi(d z, d x)
$$

it suffices to show that each of the two processes on the right hand side converges a.s. uniformly on $[0, a]$.

Hence, without loss of generality in what follows we will assume that $f=f_{1}$, i.e. $f$ is nonnegative, $t \mapsto f(t, x)$ is càdlàg and nondecreasing for any $x \in E \backslash N$ and $f$ satisfies (5.5).

Let us denote

$$
B_{a}=\{(z, y) \in \mathbb{R} \times E:|z f(a, x)| \leq 1\} .
$$

Thanks to the assumption (5.5) it is immediate to see that

$$
\int_{B_{a}^{c}} \frac{c_{\alpha}}{|z|^{1+\alpha}} d z m(d x)<\infty
$$

Hence $\pi$ has a finite number of points in $B_{a}^{c}$. It is therefore enough to consider only the part of $X^{(n)}$ which is an integral over the set $A_{n}:=\left(\left\{|z|>\delta_{n}\right\} \times E_{n}\right) \cap B_{a}$.

Denote

We will show that

$$
Y_{t}^{(n)}:=\int_{A_{n}} z f(t, x) \pi(d z, d x) .
$$

$$
\lim _{m, n \rightarrow \infty} \mathbf{E} \sup _{t \in[0, a]}\left|Y_{t}^{(n)}-Y_{t}^{(m)}\right|^{2}=0
$$

This will imply that $Y^{(n)}$ converges in probability in the supremum norm, but since $Y^{(k)}-Y^{(k-1)}, k=1,2, \ldots$ are independent we can once again use Lemma 4.4, which implies that $Y^{(n)}$ converge a.s. in the supremum norm, thus the limit is càdlàg.

Hence to complete the proof of the theorem it suffices to show (5.6). This is similar to the proof of Lemma 4.6. Suppose that $n \geqslant m$, then

$$
Y_{t}^{(n)}-Y_{t}^{(m)}=\sum_{k:\left(y_{k}, x_{k}\right) \in\left(A_{n} \backslash A_{m}\right)} \varepsilon_{k} y_{k} f\left(t, x_{k}\right)
$$

Integrating out first with respect to $\varepsilon_{i}$ and applying Theorem 1 of [3] we have that

$$
\begin{gathered}
\mathbf{E} \sup _{t \in[0, a]}\left|Y_{t}^{(n)}-Y_{t}^{(m)}\right|^{2} \leq C \mathbf{E} \sum_{k:\left(y_{k}, x_{k}\right) \in A_{n} \backslash A_{m}} y_{i}^{2} f^{2}(a, x) \\
=\int_{A_{n} \backslash A_{m}} y^{2} f^{2}(a, x) \frac{2 c_{\alpha}}{y^{\alpha+1}} d y m(d x) \rightarrow 0 .
\end{gathered}
$$

The last convergence follows from the fact that

$$
\lim _{n \rightarrow \infty} \int_{A_{n}} y^{2} f^{2}(a, x) \frac{2 c_{\alpha}}{y^{\alpha+1}} d y m(d x)=\int_{B_{a}} y^{2} f^{2}(a, x) \frac{2 c_{\alpha}}{y^{\alpha+1}} d y m(d x)
$$

which is finite by assumption (5.5). 


\section{References}

[1] Basse-O'Connor, A., Rosinski, J., On the uniform convergence of random series in Skorohod space and representations of càdlàg infinitely divisible processes. Ann. Probab. 41 (2013), no. 6, 4317-4341. MR-3161476

[2] Billingsley, P., Convergence of Probability Measures, 2nd edition 1999. MR-1700749

[3] Bednorz, W and Martynek R. A Levy-Ottaviani type inequality for the Bernoulli process on an interval, Statitics and Probab. Letters, 162 (2020). MR-4077036

[4] Davydov, Y., Dombry, C., On the convergence of LePage series in Skorokhod space. Statistics and Probab. Letters, 82 (2012), no. 1, 145-150. MR-2863036

[5] Kallenberg, O. Series of random processes without discontinuities of the second kind, Ann. Probab. 2, (1974), 729-737. MR-0370679

[6] Liu, Y. and Zhai, J., A note on time regularity of generalized Ornstein-Uhlenbeck process with cylindrical stable noise, C. R. Acad. Sci. Paris 350 (2012), 97-100. MR-2887844

[7] Liu, Y. and Zhai, J., Time Regularity of Generalized Ornstein-Uhlenbeck Processes with Lévy Noises in Hilbert Spaces. J Theor. Probab. 29 (2016), 843-866. MR-3540481

[8] Peszat, S. and Zabczyk, J., Stochastic Partial Differential Equations with Levy Noise: Evolution Equations Approach, Cambridge University Press, Cambridge, 2007. MR-2356959

[9] Peszat, S. and Zabczyk, J., Time regularity of solutions to linear equations with Lévy noise in infinite dimensions, Stochastic Proc. and App., 123, (2013), 719-751. MR-3005003

[10] Priola, E. and Zabczyk, J. Structural properties of semilinear SPDEs driven by cylindrical stable processes. PTRF,149, (2011), 97-137. MR-2773026

[11] Samorodnitsky, G. and Taqqu, M. S. Stable Non-Gaussian Random Processes. Stochastic Modelling. CRC Press, first edition, 1994. MR-1280932

Acknowledgments. We are grateful to Martin Hairer who provided a nice MR macro and to Sébastien Gouëzel for his useful comments on the internals of the class file. 


\section{Electronic Journal of Probability Electronic Communications in Probability}

\section{Advantages of publishing in EJP-ECP}

- Very high standards

- Free for authors, free for readers

- Quick publication (no backlog)

- Secure publication $\left(\mathrm{LOCKSS}^{1}\right)$

- Easy interface (EJMS²)

\section{Economical model of EJP-ECP}

- Non profit, sponsored by $\mathrm{IMS}^{3}, \mathrm{BS}^{4}$, ProjectEuclid ${ }^{5}$

- Purely electronic

\section{Help keep the journal free and vigorous}

- Donate to the IMS open access fund ${ }^{6}$ (click here to donate!)

- Submit your best articles to EJP-ECP

- Choose EJP-ECP over for-profit journals

\footnotetext{
${ }^{1}$ LOCKSS: Lots of Copies Keep Stuff Safe http://www. lockss.org/

${ }^{2}$ EJMS: Electronic Journal Management System http://www.vtex.lt/en/ejms.html

${ }^{3}$ IMS: Institute of Mathematical Statistics http://www.imstat.org/

${ }^{4}$ BS: Bernoulli Society http://www. bernoulli-society.org/

${ }^{5}$ Project Euclid: https://projecteuclid.org/

${ }^{6}$ IMS Open Access Fund: http://www.imstat.org/publications/open.htm
} 